\title{
THE ROLE OF STAVUDINE IN THE SOUTH AFRICAN PUBLIC SECTOR ANTIRETROVIRAL PROGRAMME: SHOULD THE PERFECT BE THE ENEMY OF THE GOOD?
}

\author{
Robin Wood, $B S c, B M, M M e d, F C P(S A)$ \\ Desmund Tutu HIV Centre, Institute of Infectious Disease and Molecular Medicine, University of Cape Town
}

\begin{abstract}
Stavudine (d4T) was one of the first nucleoside analogues developed as an HIV antiretroviral (ARV). An early monotherapy trial demonstrated similar antiviral activity to zidovudine (AZT), ${ }^{1}$ and a comparative study of $d 4 T$ and AZT in combination with lamivudine (3TC) and a protease inhibitor(PI) reported similar therapeutic outcome in each randomised treatment arm. ${ }^{2}$

Since registration in 1993 by the US Federal Drug Agency, d4T has been used extensively in combination therapy and was one of the first ARVs to become available in South Africa as a generic formulation. There is recently published evidence that its use is associated with higher CD4 cell count responses than other nucleoside analogues. ${ }^{3}$ The generic formulation with 3TC and nevirapine (NVP) is currently the cheapest ARV combination therapy available worldwide. With the development of a necessary public health approach to expanded access to ARVs in resource-poor settings the World Health Organization (WHO) included $\mathrm{d} 4 \mathrm{~T}$ in its recommended first-line ARV regimens. ${ }^{4}$ Following widespread use of $d 4 \mathrm{~T}$, adverse events including lipodystrophy, neuropathy and lactic acidosis associated with long-term therapy have been increasingly recognised. Despite the proven utility of $\mathrm{d} 4 \mathrm{~T}$ in more than a decade of use and its very low cost there has been an increasing swing of medical opinion against use of $d 4 T$ and a search for alternatives.
\end{abstract}

\section{MECHANISM OF ACTION}

Stavudine is a nucleoside analogue of thymidine, which is a pro-drug requiring phosphorylation by cellular kinases to the active metabolite stavudine triphosphate. The triphosphorylated molecule inhibits HIV reverse transcriptase by competing with the natural substrate deoxythymidine triphosphate and by incorporation into the viral cDNA causing chain termination. ${ }^{5}$ Resistance genotypes generated during d4T use show much overlap with those generated by AZT, another thymidine analogue. D4T also inhibits human cellular DNA polymerases beta and gamma resulting in a marked reduction in the synthesis of mitochondrial DNA. This crossinhibition of human DNA polymerases may constitute the causative mechanism of the more serious toxicities associated with d4T use.

\section{TOXICITY}

The toxicity of $d 4 T$ is exacerbated when the drug is used in combination with other dideoxy nucleoside analogues and the combination with didanosine (ddl) is now discouraged in most treatment guidelines. Impairment of mitochondrial function is postulated to be the cause of increased lactate production and/or decreased clearance. The clinical sequelae vary from asymptomatic hyperlactataemia to fatal lactic acidosis, and from hepatomegaly with steatosis to hepatic failure. Recognised risk factors for lactic acidosis include female gender, increased body mass index, and prolonged use of d4T. Monitoring for elevated lactate is indicated in patients on $\mathrm{d} 4 \mathrm{~T}$ when hepatic transaminases rise, or there is unexplained weight loss or gastrointestinal symptoms. Peripheral neuropathy is also considered to result from mitochondrial dysfunction and symptoms are related to the dose of $d 4$ T. Neuropathy is more frequently reported in patients with advanced HIV disease and those with a prior history of neuropathy and when other neurotoxic drugs such as isoniazid and ddl are coadministered.

\section{EXPERIENCE WITH $d 4$ T IN SOUTH AFRICAN PUBLIC} SECTOR PROGRAMMES

The Cape Town Gugulethu treatment programme ${ }^{6}$ was the first public sector ARV programme to initiate therapy in September 2002 with d4T/3TC and a non-nucleoside reverse transcriptase inhibitor (NNRTI) using a treatment protocol based on the WHO 2002 expanded access recommendations. ${ }^{4}$ A second regimen of AZT, ddl and ritonavir-boosted lopinavir (Kaletra) is available to those who fail first-line therapy. Mortality early in this programme showed that $66 \%$ of deaths 
occurred in patients awaiting ART and the majority of deaths on ART occurred in the first 6 months of treatment due to causes associated with the advanced stage of HIV infection of those accessing treatment. ${ }^{7}$ Of the 68 programme deaths only 2 were ARV drug-related, a nevirapine rash with septicaemia and a d4T-associated lactic acidosis. ${ }^{7}$ By April 2006 over 2000 patients had received ARVs in this clinic. D4T substitution has been required in 206 individuals in the treatment programme, in 158 cases as a direct result of drug toxicity. The median time on d4T before switching was 403 days (IQR 280 - 569). Causes of drug-related switches were, in descending frequency, lipodystrophy (76 cases, 48\%), peripheral neuropathy (57, $36 \%)$, hyperlactataemia (16, with 8 cases of acidosis - 10\% and $5 \%$ respectively), elevated hepatic transaminases $(5,3 \%)$, unspecified causes $(3,2 \%)$ and pancreatitis $(1,1 \%)$. Another large HIV treatment programme in Khayelitsha, Cape Town, which began using d4T as a first-line regimen in 2003, has reported approximately $10 \%$ of patients switching from both AZT and d4T by 12 months. ${ }^{8}$ Nucleoside reverse transcriptase inhibitor (NRTI) switches due to AZT toxicity were mainly the result of anaemia (82\%), which occurred early, while d4T switches were reported later in therapy and appeared to be increasing with longer use of $\mathrm{d} 4 \mathrm{~T}$ in the programme. These cohort data indicate a higher rate of switching due to $d 4 T$ toxicity than is reflected in published randomised trial data. A multinational comparative 3-year study of 602 drug-naïve individuals (26\% female and 20\% black) randomised to d4T or tenofovir in combination with 3TC and efavirenz had identical discontinuation rates (6\%) due to adverse events in each drug allocation arm, and a sub-analysis of women in the study also showed similar regimen discontinuation rates in both arms. ${ }^{9}$ Lactic acidaemia was reported in 1 patient who had been randomised to the $\mathrm{d} 4 \mathrm{~T}$ arm. If similar dosages and formulations of $d 4 \mathrm{~T}$ are used in Gugulethu and Khayelitsha, there are two possible explanations for this apparent increased switching due to toxicity: firstly that the South African population is more susceptible to adverse effects of $d 4 T$ than the randomised study population, or that there is significant ascertainment bias in the cohort data due to over-diagnosis of possible d4T-related clinical events. It is particularly important to establish if there is an increased population susceptibility to adverse effects of $d 4 \mathrm{~T}$, which may in turn be due to genetic or exposure to environmental cofactors.

\section{ROLE OF (d4T) IN TREATMENT PROGRAMMES}

d4T has been used successfully in the public health ARV programmes because it is cheap and initially very well tolerated. Toxicities accumulate after prolonged therapy and pose significant challenges to programme staff, who must maintain a high vigilance for potentially serious metabolic complications. The inability to monitor serum lactate easily together with the serious consequences that can result makes management of lactate metabolic derangements particularly problematic.

However, the major challenge facing the South African ARV programme is to reduce HIV-related deaths by increasing coverage of the existing programme to the 800000 individuals currently in immediate need of therapy. The public health approach is epitomised by national tuberculosis control programmes, where treatment regimens are cheap, simple and effective and co-formulations are used where possible. Treatment options are limited, so that medical officers and nurses can be well acquainted with a small number of agents. First-line ARV therapy should be well tolerated and sideeffects should be predictable and require minimal toxicity monitoring. Within such a public health framework it is important to establish whether any changes to existing regimens or use of alternative therapies will help or impede the necessary wider access to ARV therapy.

\section{POSSIBLE ALTERNATIVE REGIMENS}

Standard ARV therapy is based on a nucleoside backbone of two NRTIs together with either an NNRTI or a PI. There are 6 NRTIs registered in South Africa, stavudine (d4T), zidovudine (AZT), lamivudine (3TC), didanosine (ddl), zalcitabine (ddC) and abacavir $(A B C)$. Of these only $d d C$ and $A B C$ are not already included in standard first- and second-line regimens. Currently the cost of $A B C$ and the neurotoxicity of $d d C$ would exclude them as substitutes for $d 4 T$ in a first-line regimen. With the present choice of registered NRTIs, substitution of AZT for d4T in the initial first-line regimen would necessitate the subsequent use of $d 4 \mathrm{~T}$ with $\mathrm{ddl}$ in the second-line regimen, a combination that is discouraged due to co-toxicity. The present use of AZT in second-line following initial d4T failure in first-line therapy is not ideal, as both drugs have shared HIV resistance mutation profiles.

The choice of available NRTls will be increased if tenofovir (TNF), a nucleotide analogue of adenosine 5'-monophosphate, is registered for use in South Africa. The drug has been licensed since 2001 in the USA and is under review for registration by the South African Medicines Control Council. Its US labelled use was initially for patients failing previous therapies and more recently extended to use in first-line therapy. It is generally well tolerated with some gastrointestinal adverse events and increased bioavailability when administered with food (40\%). The prolonged elimination half-life allows oncedaily administration, with the major route of elimination being both renal glomerular filtration and active tubular secretion. It has adverse pharmacokinetic interactions with $\mathrm{ddl}$ and has activity against hepatitis $B$ virus which may precipitate severe acute exacerbations of hepatitis $B$ in patients who have discontinued the drug. ${ }^{10}$ The use of TNF, which will require additional renal function monitoring, will increase the programme costs of safety monitoring. TNF is also coformulated with emitricitabine (FTC) into a single daily tablet (Truvada), which offers a reduced pill burden.

If TNF became available for use in the national ARV programme in South Africa, how could it be utilised? Substitution of d4T with TNF would increase the cost of the first-line regimen and increase monitoring requirements and therefore impede increased access to therapy. The cost 
effectiveness of an ARV programme is very sensitive to the costs of the first-line therapy. ${ }^{11} A$ direct substitution of the AZT in the second-line regimen would not be possible as coadministration with $\mathrm{ddl}$ is discouraged. Replacement of both AZT and ddl with TNF and 3TC or FC would not increase costs and would provide a more rational second-line NRTI backbone. This strategy, although it would require the recycling of $3 T C / F T C$, would release AZT to be used as a switch alternative for $\mathrm{d} 4 \mathrm{~T}$ within the first-line regimen. The toxicity profiles of AZT and d4T differ in both spectrum and timing (Table I). Anaemia related to AZT occurs early after initiation of treatment in approximately 6 - 10\% of African patients, while $\mathrm{d} 4 \mathrm{~T}$ is initially well tolerated. D4T and AZT could be used in a similar fashion to efavirenz and nevirapine, which have different toxicity profiles but are susceptible to similar genetic mutations of HIV. The use of low-cost d4T/3TC/NVP could be maintained in the initial first-line regimen if either those identified at high risk or those with early d4T toxicity could be switched to AZT.

\section{SUMMARY}

The major challenge facing the ARV programme in South Africa is to expand access rapidly to very large numbers of individuals at high immediate risk of death. In order to achieve this, a public health approach to ARV therapy requires the use of cheap effective drugs in simply administered regimens. Currently the generic combination of $d 4 T / 3 T C / N V P$ constitutes the cheapest available ARV regimen. Serious toxicities associated with long-term use of $\mathrm{d} 4 \mathrm{~T}$ have raised concerns about the continued use of this drug in the first-line regimen. The rate of drug switching due to d4T toxicity in South African cohorts appears higher than that reported in controlled randomised studies, and this may either be due to a real higher toxicity event rate in the South African population or be an apparent increase caused by ascertainment biases. Mortality in existing ARV programmes is overwhelmingly dominated by late access to programmes rather than deaths due to drug toxicity. Changes to present ARV regimens must therefore be judged within a public health framework, which should enable wider access to therapy for those in immediate need of treatment.

\section{REFERENCES}

1. Spruance SL, Pavia AT, Mellors JW, et al. Clinical efficacy of monotherapy with stavudine compared with zidovudine in HIV-infected, zidovudine-experienced
TABLE I. COMPARISON OF RAND COSTS PER MONTH (PUBLIC SECTOR TENDER PRICES MAY 2006), TOXICITIES, AVAILABLE CO-FORMULATIONS AND PILL BURDEN OF STAVUDINE, ZIDOVUDINE, ABACAVIR AND TENOFOVIR

\begin{tabular}{|lcccc|}
\hline & $\begin{array}{c}\text { Stavudine } \\
\text { (d4T) }\end{array}$ & $\begin{array}{c}\text { Zidovudine } \\
\text { (AZT) }\end{array}$ & $\begin{array}{c}\text { Abacavir } \\
\text { (ABC) }\end{array}$ & $\begin{array}{c}\text { Tenofovir } \\
\text { (TNF) }\end{array}$ \\
\hline $\begin{array}{l}\text { Cost/ } \\
\text { month } \\
\text { (generic) }\end{array}$ & $\begin{array}{l}\text { R42.59 } \\
\text { (22.66) }\end{array}$ & $\begin{array}{c}\text { R 126.79 } \\
(143.64)\end{array}$ & $\begin{array}{c}\text { R527.92 } \\
\text { (NA) }\end{array}$ & $\begin{array}{c}\text { R123.05 } \\
\text { (NA) }\end{array}$ \\
$\begin{array}{l}\text { Toxicity } \\
\text { timing }\end{array}$ & Late & Early & Early & Early \\
$\begin{array}{l}\text { Main } \\
\text { toxicities }\end{array}$ & $\begin{array}{l}\text { Leuropathy } \\
\text { Lactic acidosis }\end{array}$ & Anaemia & Hypersensitivity & Renal \\
& Lipodystrophy & & & tubular \\
$\begin{array}{l}\text { Available } \\
\text { combina- } \\
\text { tions }\end{array}$ & d4T/3TC/NVP & $\begin{array}{c}\text { AZT/3TC; } \\
\text { AZT/3TC/ABC }\end{array}$ & AZT/3TC/ABC & TNF/FTC \\
$\begin{array}{l}\text { Pill burden/ } \\
\text { day }\end{array}$ & 2 tabs & 2 tabs & 2 tabs & 1 tab \\
\hline & & & \\
\hline
\end{tabular}

patients. A randomized, double-blind controlled trial. Bristol-Myers Squibb/019 Study Group. Ann Intern Med 1997; 126(5): 355-363.

2. Squires $K E$, Gulick $R$, Tebas $P$, et al. A comparison of stavudine plus lamivudine versus zidovudine plus lamivudine in combination with indinavir in antiretroviral naïve individuals with HIV infection: selection of thymidine analog regimen therapy (START 1). AIDS 2000; 14(11): 1591-1600.

3. Mocroft A, Phillps AN, Lederberber B, et al. Relationship between antiretrovirals used as part of a CART regimen and CD4 cell count increases in patients with suppressed viremia. AIDS 2006; 20(8): 1141-1150.

4. World Health Organization. Scaling up antiretroviral therapy in resourcelimited settings: Guidelines for a public health approach. World Health Organization, Executive Summary April 2002, Geneva, Switzerland. http://www.who.int/hiv/topics/arv/en/scaling_exe_summary.pdf (accessed 14 June 2006).

5. Zerit (stavudine) patient information leaflet. Bristol-Myers Squibb. Princeton NJ 08543, USA. http://bms.com (accessed 16 June 2006).

6. Bekker L-G, Myer L, Orrell C, Wood R. A South African community-based antiretroviral programme: Outcomes during 3 years of scale-up. S Afr Med J 2006; 96(4): 315-320

7. Lawn SD, Myer L, Orrel C, Bekker L-G, Wood R. Early mortality among patients accessing a community-based antiretroviral service in South Africa: Implications for program design. AIDS 2005; 19: 2141-2148.

8. Boule $A$, Hildebrand $K$, Coetzee $D$, et al. Regimen durability and reasons for regimen changes in the first 1000 treatment naive adults accessing ART in Kayelitsha. 9th International Workshop on HIV Databases, Budapest, Hungary, 21-22 April 2005.

9. Gallant JE, Staszewski S, Pozniac AL, et al. Efficacy and safety of tenofovir vs stavudine in combination therapy in antiretroviral-naïve patients: a 3-year randomized trial. JAMA 2004; 292(2): 266-268.

10. Truvada patient information leaflet. http://www.gilead.com/pdf/truvada pi/pdf (accessed 16 June 2006).

11. Badri M, Cleary S, Maartens $G$, et al. When to initiate HAART in sub-Saharan Africa: a cost-effectiveness study. Antivir Ther 2006: 11(1): 63-72. 